\title{
Using interaction-based readouts to approach the ultimate limit of detection noise robustness for quantum-enhanced metrology in collective spin systems
}

Simon A. Haine, Ruvi Lecamwasam, Joseph Hope

Simon A. Haine, Ruvi Lecamwasam, Joseph Hope, "Using interaction-based readouts to approach the ultimate limit of detection noise robustness for quantum-enhanced metrology in collective spin systems," Proc. SPIE 10934, Optical, Opto-Atomic, and Entanglement-Enhanced Precision Metrology, 109341N (1 March 2019); doi: 10.1117/12.2511835

SPIE. Event: SPIE OPTO, 2019, San Francisco, California, United States 


\title{
Using Interaction-Based Readouts to Approach the Ultimate Limit of Detection Noise Robustness for Quantum-Enhanced Metrology in Collective Spin Systems.
}

\author{
Simon A. Haine ${ }^{1}$, Ruvi Lecamwasam ${ }^{1,2}$, and Joseph Hope ${ }^{1}$ \\ ${ }^{1}$ Department of Quantum Science, Australian National University, Canberra, Australia \\ ${ }^{2}$ Australian Research Council Centre for Quantum Computing and Communication Technology
}

\begin{abstract}
We consider the role of detection noise in quantum-enhanced metrology in collective spin systems, and derive a fundamental bound for the maximum obtainable sensitivity for a given level of added detection noise. We then present an interaction-based readout utilising the commonly used one-axis twisting scheme that approaches this bound for states generated via several commonly considered methods of generating quantum enhancement, such as one-axis twisting, two-axis counter-twisting, twist-and-turn squeezing, quantum non-demolition measurements, and adiabatically scanning through a quantum phase transition. We demonstrate that this method performs significantly better than other recently proposed interaction-based readouts. These results may help provide improved sensitivity for quantum sensing devices in the presence of unavoidable detection noise.
\end{abstract}

Keywords: Quantum Metrology, Atom Interferometry

\section{INTRODUCTION}

There is a continued push for improved metrological potential in devices such as atomic clocks, atomic magnetometers, and inertial sensors based on atom interferometry, ${ }^{1}$ the physics of which are well described by collective spin-systems. ${ }^{2}$ Over the last decade there has been rapid progress in the demonstration of quantum enhanced metrology in these systems, that is, parameter estimation with sensitivity surpassing the shot-noise limit (SNL) ${ }^{3-19}$ These schemes generally require a state preparation step, where inter-particle entanglement is created to enhance the metrological potential, ${ }^{20-22}$ before the classical parameter of interest (which is usually proportional to a phase) is encoded onto the state and then measured. There exist a plethora of state preparation techniques for creating quantum enhanced states, such as quantum state transfer from light to atoms, ${ }^{23-32}$ quantum non-demolition measurements (QNDs) ${ }^{4,18,33-36}$ spin changing collisions, ${ }^{10,11,37-39}$ one-axis twisting $(\mathrm{OAT}),{ }^{3,6,8,9,40-42}$ two-axis counter-twisting (TACT), ${ }^{40,43}$ twist-and-turn squeezing (TNT), ${ }^{16,44}$ and adiabatically scanning through a quantum phase transition (QPT). ${ }^{45-51}$ In order to see significant quantum enhancement however, we almost always require detection with very low noise, of the order of less than one particle. ${ }^{2,52,53}$

Recent progress indicates that this can be relaxed via the concept of interaction-based readouts (IBRs), ${ }^{50,51,54-67}$ where periods of unitary evolution are applied to the system after the phase encoding step, but before the measurement takes place. These readouts usually involve inter-particle interactions, similar to the ones used for the state preparation. Davis et al. showed that by using OAT to prepare a state with high quantum Fisher information (QFI) before applying a phase shift, and then employing an IBR that reverses the OAT dynamics, quantum enhanced sensitivity could be achieved well beyond the Gaussian spin-squeezing regime. Furthermore, this quantum enhancement persisted even when the added detection noise was as large as the projection noise. ${ }^{54}$ Similarly, Hosten et al. experimentally demonstrated that a period of nonlinear evolution after the state preparation and phase encoding could achieve sub-SNL sensitivity even in the presence of significant detection noise. ${ }^{55}$ Macri et al. demonstrated that by performing an IBR that perfectly reverses the state preparation and then projects into the initial state, the sensitivity saturates the quantum Cramér-Rao bound (QCRB). ${ }^{57}$ Nolan et $a l .{ }^{60}$ further generalised this result to show that there exist many IBRs that satisfy the conditions for saturating the QCRB, and that the choice of IBR has implications for the level of sensitivity in the presence of detection

simon.a.haine@gmail.com

Optical, Opto-Atomic, and Entanglement-Enhanced Precision Metrology, edited by Selim M. Shahriar, Jacob Scheuer, Proc. of SPIE Vol. 10934, 109341N · (c) 2019 SPIE · CCC code: $0277-786 X / 19 / \$ 18 \cdot$ doi: $10.1117 / 12.2511835$ 
noise (or "robustness"). In particular, it was found that the optimum IBR was not necessarily the one that perfectly reversed the state preparation. Furthermore, sensitivity approaching the Heisenberg limit ${ }^{68,69}$ could be achieved in the presence of detection noise approaching the number of particles. IBRs have also been explored by applying time-reversal of the state-preparation dynamics in systems where the quantum-enhanced state is generated via SCC, ${ }^{58,59,70} \mathrm{TACT},{ }^{62} \mathrm{TNT}^{64}$ and QPT. ${ }^{50,51}$

In this work, we derive a limit for sensitivity in the presence of detection noise which is significantly better than the levels achievable via previous schemes. We then present an IBR based on OAT that approaches this limit for states generated via OAT, TNT, TACT, QPT, and QND.

\section{ULTIMATE SENSITIVITY LIMIT IN THE PRESENCE OF DETECTION NOISE}

Suppose we perform a POVM measurement on a quantum state $\rho_{\theta}$ which depends on some classical parameter $\theta$. This will be represented by a probability distribution $P_{m}$, where $m$ denotes the measurement result. The sensitivity with which we can estimate $\phi$ by sampling $P_{m}$ is quantified via the Cramér-Rao bound: $\Delta \phi^{2}=$ $1 /\left(n F_{C}\right)$, where $n$ is the number of measurements, $F_{C}$ is the classical Fisher information (CFI) defined by

$$
F_{C}=\sum_{m} \dot{P}_{m}^{2} / P_{m}
$$

and $\dot{P}_{m} \equiv \partial_{\phi} P_{m}$. Assuming a collection of $N$ particles distributed amongst two modes, the natural description for our system is provided via the pseudo-spin $\mathrm{SU}(2)$ algebra: $\left[\hat{J}_{x}, \hat{J}_{y}\right]=i \hat{J}_{z} \cdot{ }^{71}$ The eigenstates of these operators form a natural basis of easily accessible measurements, as they can be obtained via single-particle operations such as linear rotations and particle counting. ${ }^{2}$ For simplicity, throughout this paper we assume that measurements are made by projecting into the $\hat{J}_{z}$ basis, i.e. , $\{|m\rangle\langle m|\}$, where $\hat{J}_{z}|m\rangle=m|m\rangle$. The particular direction is of little consequence, however, as projections along other directions can be obtained via linear rotations. Following the convention introduced in ${ }^{72}$ and subsequently used in, ${ }^{2,50,56,60,62,64,65,70}$ we model the behaviour of an imperfect detector as sampling from the probability distribution

$$
\tilde{P}_{m}(\sigma)=\sum_{m^{\prime}} \Gamma_{m, m^{\prime}}(\sigma) P_{m^{\prime}}
$$

where

$$
\Gamma_{m, m^{\prime}}(\sigma)=e^{-\left(m-m^{\prime}\right)^{2} /\left(2 \sigma^{2}\right)} / \sum_{m} e^{-\left(m-m^{\prime}\right)^{2} /\left(2 \sigma^{2}\right)}
$$

introduces detection noise of magnitude $\sigma$. This is equivalent to the positive-operator valued measurement (POVM) $\left\{\hat{M}_{m}\right\}=\left\{\sum_{m^{\prime}} \Gamma_{m, m^{\prime}}\left|m^{\prime}\right\rangle\left\langle m^{\prime}\right|\right\}$. To demonstrate how this noise affects the CFI, we consider the case where $P_{m}$ contains only two non-zero elements, $P_{a}$ and $P_{b}$, with $P_{b}=1-P_{a}$, and $\dot{P}_{a}=-\dot{P}_{b}=\sqrt{F_{0}\left(P_{a}-P_{a}^{2}\right)}$, such that $F_{C}=F_{0}$. We can obtain an approximate expression $\tilde{P}_{m}$ by approximating $m$ as a continuous variable such that

$$
P(m)=P_{a} \delta(m-a)+P_{b} \delta(m-b)
$$

and

$$
\Gamma\left(m-m^{\prime}\right)=\frac{1}{\sigma \sqrt{2 \pi}} e^{-\left(m-m^{\prime}\right)^{2} /\left(2 \sigma^{2}\right)} .
$$

Using these in Eq. (2), we find

$$
\begin{aligned}
\tilde{P}(m) & =\int_{-\infty}^{\infty} \Gamma\left(m-m^{\prime}\right) P\left(m^{\prime}\right) d m \\
& =\left(P_{a} e^{-(a-m)^{2} / 2 \sigma^{2}}+P_{b} e^{-(b-m)^{2} / 2 \sigma^{2}}\right) / \sqrt{2 \pi} \sigma
\end{aligned}
$$

Defining

$$
\begin{aligned}
\tilde{P}_{a} & =\int_{-\infty}^{\frac{1}{2}(a+b)} \tilde{P}(m) d m \\
& =\frac{1}{2}\left(1+\left(2 P_{a}-1\right) \operatorname{Erf}\left[\frac{b-a}{2 \sqrt{2} \sigma}\right]\right),
\end{aligned}
$$


and

$$
\begin{aligned}
\tilde{P}_{b} & =\int_{\frac{1}{2}(a+b)}^{\infty} \tilde{P}(m) d m \\
& =\frac{1}{2}\left(1+\left(1-2 P_{a}\right) \operatorname{Erf}\left[\frac{b-a}{2 \sqrt{2} \sigma}\right]\right),
\end{aligned}
$$

(assuming $a<b$ ), and maximising with respect to $P_{a}\left(P_{a} \rightarrow P_{b} \rightarrow \frac{1}{2}\right)$ we obtain

$$
F_{C}(\sigma)=\dot{\tilde{P}}_{a}^{2} / \tilde{P}_{a}+\dot{\tilde{P}}_{b}^{2} / \tilde{P}_{b} \approx F_{0}(\operatorname{Erf}[(a-b) / 2 \sqrt{2} \sigma])^{2} .
$$

Clearly, $F_{C}(\sigma)$ decays less rapidly when the separation between the non-zero components of $P_{m},|a-b|$, is large compared to $\sigma$. This intuition leads us to postulate that distribution with maximum robustness, $P_{\mathrm{opt}}$ is

$$
\begin{aligned}
& P_{N / 2}=P_{-N / 2}=\frac{1}{2}, \\
& \dot{P}_{N / 2}=-\dot{P}_{-N / 2}=\sqrt{F_{0}} / 2,
\end{aligned}
$$

with all other elements equal to zero. In the absence of detection noise, the QCRB states that $F_{C} \leq F_{Q}$, where $F_{Q}$ is the QFI. We define the noisy $Q C R B$ (NQCRB) as $F_{C}(\sigma) \leq F_{n}(\sigma)$, where $F_{n}(\sigma)$ is the CFI calculated from the $\left\{\tilde{P}_{m}(\sigma), \dot{\tilde{P}}_{m}(\sigma)\right\}$ obtained from performing the discrete sum in Eq. (2) numerically with $\left\{P_{m}, \dot{P}_{m}\right\}=\left\{P_{\text {opt }}, \dot{P}_{\text {opt }}\right\}$, and setting $F_{0}=F_{Q}$. This is the maximum sensitivity that can be achieved by making spin measurements on a state with QFI equal to $F_{Q}$ in the presence of detection noise $\sigma$. We can get an approximate analytic expression for $F_{n}(\sigma)$ by again approximating $m$ as a continuous variable, but limiting the range to $-N / 2<m<N / 2$, such that

$$
F_{n}(\sigma) \approx F_{Q}\left(1-2 \frac{\operatorname{Erf}[\alpha / 2]}{\operatorname{Erf}[\alpha]}\right)^{2}
$$

with $\alpha=N / \sqrt{2} \sigma$.

As an aside, note that we can derive an exact analytical form for $F_{n}(\sigma)$ in arbitrary dimensions $N$ in a number of ways. The first is to explicitly calculate the probability distribution $\tilde{P}_{m}(\sigma)$ and then use the explicit formula Eq. (1). We can gain more insight however by looking at this problem through the lens of information geometry. ${ }^{73,74}$ If there are $M$ possible measurement outcomes, we can imagine $P_{m}$ as a point in the positive octant of $\mathbb{R}^{m}$ satisfying $P_{1}+\cdots+P_{M}=1$. As the parameter $\phi$ varies, we move along a curve of probability distributions in this subset. Intuitively we would expect the speed at which we move along this curve to correspond to the CFI, as a high speed means that a small change in $\phi$ gives us a very different state, however there is no geometric meaning to the tangent vector $\dot{P}_{1}^{2}+\cdots+\dot{P}_{m}^{2}$.

It is in fact more natural to define $\wp_{m}=\sqrt{P_{m}}$. In this case the manifold of probability distributions corresponds to the unit sphere $\wp_{1}^{2}+\cdots+\wp_{M}^{2}$ in $\mathbb{R}^{M}$, and we can verify that

$$
F_{C}=\sum_{m} \dot{P}_{m}^{2} / P_{m}=4 \sum_{m} \dot{\wp}_{m}^{2} .
$$

If we now look at $\wp_{m}$ as a curve on the unit sphere parameterised by $\phi$, the CFI corresponds to the magnitude of the tangent vector of this curve. Moreover, we can now apply the powerful machinery of differential geometry to gain new insights and techniques of proof. Defining $\tilde{\wp}_{m}(\sigma)=\sqrt{\tilde{P}_{m}(\sigma)}$, we can denote as $\Lambda(\sigma)$ the error mapping which sends $\wp_{m} \rightarrow \tilde{\wp}_{m}(\sigma)$. The CFI after error will be given by the tangent vector of the $\tilde{\wp}_{m}(\sigma)$ curve, which can be found by applying the pushforward of $\Lambda(\sigma)^{75}$ to the tangent vector of $\wp_{m}$. Using this we can derive that for the probability distribution in Eq. (10), the tangent vector with error is

$$
\dot{\tilde{\wp}}_{m}=\frac{1}{2 \sqrt{2 A(\sigma)}} \frac{e^{-\frac{(m-N / 2)^{2}}{2 \sigma^{2}}}-e^{-\frac{(m+N / 2)^{2}}{2 \sigma^{2}}}}{\sqrt{e^{-\frac{(m-N / 2)^{2}}{2 \sigma^{2}}}+e^{-\frac{(m+N / 2)^{2}}{2 \sigma^{2}}}}},
$$




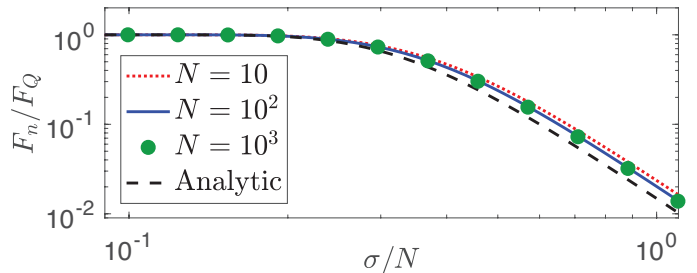

Figure 1. The exact value of $F_{n}$ vs. $\sigma / N$ (Eq. (14)) for $N=10,10^{2}$ and $10^{3}$, compared to the approximate expression Eq. (11). The shape of $F_{n}(\sigma / N)$ is almost identical for $N=10^{2}$ and $10^{3}$.

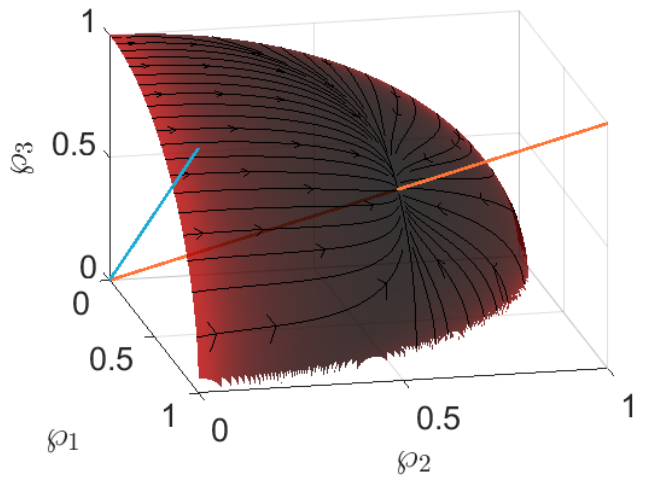

Figure 2. How the set of distributions $\wp_{m}$ transform under the error map $\Lambda(\sigma)$ for $M=3$. The arrows denote the direction the distribution is pulled in for $\sigma=0.3$, and the colour denotes the normalised distance $\Lambda(\sigma)$ pulls $\wp_{m}$, with red being maximum and black zero. The orange line shows the maximally entropic distribution $\wp_{m}=\frac{1}{\sqrt{3}}$ for all $m$, and the blue line shows the optimum state Eq. (10). We see that close to the optimum distribution the $\wp_{m}$ are pulled approximately parallel and the distance between points remains constant, showing how the Fisher information is conserved.

with normalisation $A(\sigma)=\sum_{k=-n / 2}^{n / 2} e^{-\frac{(n / 2-k)^{2}}{2 \sigma^{2}}}$. The exact expression corresponding to the approximation Eq. (11) is then given by

$$
F_{n}(\sigma)=4 \sum_{m} \dot{\wp}_{m}^{2}=4 \sum_{m} \frac{1}{2 \sqrt{2 A(\sigma)}} \frac{e^{-\frac{(m-N / 2)^{2}}{2 \sigma^{2}}}-e^{-\frac{(m+N / 2)^{2}}{2 \sigma^{2}}}}{\sqrt{e^{-\frac{(m-N / 2)^{2}}{2 \sigma^{2}}}+e^{-\frac{(m+N / 2)^{2}}{2 \sigma^{2}}}}}
$$

Fig.(1) shows excellent agreement between Eq. (11) and Eq. (14). Eq. (11) provides a slight under-estimate of the CFI, as information is lost when condensing $P_{m}$ into a binary distribution via Eq. (7) and Eq. (8). For the remainder of this paper, we use the exact expression (Eq. (14)) rather than Eq. (11).

We can also use these techniques to visualise how different IBR schemes behave under detector error. In the neighbourhood of some $\phi_{0}$, an IBR is represented as two nearby probability distributions $\left\{\wp_{m}\right\}_{\phi_{0}}$ and $\left\{\wp_{m}\right\}_{\phi_{0}+d \phi}$ on the positive octant of the unit sphere in $\mathbb{R}^{N}$. Under the error map $\Lambda(\sigma)$ the distance between these points will decrease, corresponding to a drop in the Fisher information. We plot in Fig. (2) the case for $N=3$. We can see that for the optimum state Eq. (10) the error map keeps the probability distributions approximately parallel, preserving their distance and hence the Fisher information.

\section{INTERACTION-BASED READOUT TO SATURATE THE QCRB}

We first review the conditions under which an IBR will saturate the QCRB. ${ }^{60,64}$ Starting with an arbitrary initial pure state $\left|\psi_{1}\right\rangle$, we note that this may always be written as $\left|\psi_{1}\right\rangle=U_{1}\left|\psi_{0}\right\rangle$, where $\left|\psi_{0}\right\rangle=\left|\frac{N}{2}\right\rangle$ is the maximal $\hat{J}_{z}$ eigenstate, which is completely separable in the particle basis. In most quantum enhanced metrology schemes the unitary operator $U_{1}$ implements the state preparation step, which may be employed to increase the QFI of an initially separable state. Specific examples of this process including OAT, TACT, TNT, and QPT will be considered later. The phase shift $\phi$ is then encoded onto the state via $\left|\psi_{\phi}\right\rangle=e^{i \hat{J}_{n} \phi}\left|\psi_{1}\right\rangle \equiv U_{\phi}\left|\psi_{1}\right\rangle$, where 
$\hat{J}_{n}=\mathbf{J} \cdot \mathbf{n}$, and $\mathbf{n}$ is a unit vector chosen to maximise the QFI of $\left|\psi_{\phi}\right\rangle$. This vector can be obtained from the collective covariance matrix. ${ }^{20}$ An IBR is some unitary $U_{2}$ such that measurements are made on the state $U_{2}\left|\psi_{\phi}\right\rangle$. Our goal is to find $U_{2}$ such that the probability distribution $P_{m}=\left|\left\langle m\left|U_{2}\right| \psi_{\phi}\right\rangle\right|^{2}$ saturates the QCRB. Writing

$$
P_{m}=\left\langle m^{\prime}\left|U_{\phi} \rho U_{\phi}^{\dagger}\right| m^{\prime}\right\rangle
$$

where

$$
\left|m^{\prime}\right\rangle=U_{2}^{\dagger}|m\rangle
$$

and

$$
\rho=\left|\psi_{1}\right\rangle\left\langle\psi_{1}\right|,
$$

for small values of $\phi$, the CFI can be calculated as the leading term in the expansion of the Hellinger statistical distance:

$$
\begin{aligned}
d_{H}^{2}(0, \phi) & =1-\sum_{m} \sqrt{P_{m}(0) P_{m}(\phi)} \\
& =F_{c}(0) \phi^{2} / 8+\mathcal{O}\left(\phi^{3}\right)
\end{aligned}
$$

For small $\phi$, Taylor expanding the probability amplitude gives

$$
P_{m}(\phi)=P_{m}(0)+\left.\frac{\partial P_{m}(\phi)}{\partial \phi}\right|_{\phi=0} \phi+\left.\frac{\partial_{\phi}^{2} P_{m}(\phi)}{\partial \phi^{2}}\right|_{\phi=0} \frac{\phi^{2}}{2}+\mathcal{O}\left(\phi^{3}\right) .
$$

Using

$$
\begin{aligned}
P_{m}(\phi) & =\left\langle m\left|U_{\phi}^{\dagger} \rho U_{\phi}\right| m\right\rangle, \\
\frac{\partial P_{m}(\phi)}{\partial \phi} & =i\left\langle m\left|\hat{J}_{n} U_{\phi} \rho U_{\phi}^{\dagger}\right| m\right\rangle+c . c . \\
\frac{\partial^{2} P_{m}(\phi)}{\partial \phi^{2}} & =\left\langle m\left|\hat{J}_{n} U_{\phi} \rho U_{\phi}^{\dagger} \hat{J}_{n}\right| m\right\rangle-\left(\left\langle m\left|\hat{J}_{n}^{2} U_{\phi} \rho U_{\phi}^{\dagger}\right| m\right\rangle+\text { c.c. }\right),
\end{aligned}
$$

we find

$$
\begin{aligned}
P_{m}(0) & =\left\langle m^{\prime}|\rho| m^{\prime}\right\rangle \\
\left.\frac{\partial P_{m}(\phi)}{\partial \phi}\right|_{\phi=0} & =i\left\langle m^{\prime}\left|\hat{J}_{n} \rho\right| m^{\prime}\right\rangle+\text { c.c. } \\
\left.\frac{\partial^{2} P_{m}(\phi)}{\partial \phi^{2}}\right|_{\phi=0} & =\left\langle m^{\prime}\left|\hat{J}_{n} \rho \hat{J}_{n}\right| m^{\prime}\right\rangle-\left(\left\langle m^{\prime}\left|\hat{J}_{n}^{2} \rho\right| m^{\prime}\right\rangle+\text { c.c. }\right)
\end{aligned}
$$

We now introduce the conditions under which our measurement saturates the QCRB:

1. The initial state is an eigenstate of the parity operator $\hat{\Pi}=\sum_{m}(-1)^{m^{\prime}}\left|m^{\prime}\right\rangle\left\langle m^{\prime}\right|$ such that $\hat{\Pi} \rho=(-1)^{p} \rho$ for $p=0,1$.

2. The generator of phase flips the parity: $\hat{\Pi} \hat{J}_{n} \hat{\Pi}=-\hat{J}_{n}$.

Under these two conditions,

$$
\begin{aligned}
\left\langle m^{\prime}|\rho| m^{\prime}\right\rangle & =(-1)^{m^{\prime}+p}\left\langle m^{\prime}|\rho| m^{\prime}\right\rangle \\
\left\langle m^{\prime}\left|\rho \hat{J}_{n}\right| m^{\prime}\right\rangle & =0 \\
\left\langle m^{\prime}\left|\hat{J}_{n} \rho \hat{J}_{n}\right| m^{\prime}\right\rangle & =(-1)^{m^{\prime}+p+1}\left\langle m^{\prime}\left|\hat{J}_{n} \rho \hat{J}_{n}\right| m^{\prime}\right\rangle
\end{aligned}
$$

which also yields

$$
P_{m}(0)\left\langle m^{\prime}\left|\hat{J}_{n} \rho \hat{J}_{n}\right| m^{\prime}\right\rangle=0,
$$


as $P_{m}(0)=0$ if $m^{\prime}+p$ is odd, and $\left\langle m^{\prime}\left|\hat{J}_{n} \rho \hat{J}_{n}\right| m^{\prime}\right\rangle=0$ if $m^{\prime}+p$ is even. After using Eq. (19) and Eq. (21) in Eq. (18), we obtain

$$
d_{H}^{2}=1-\sum_{m} \sqrt{P_{m}^{2}(0)-\frac{\phi^{2}}{2} P_{m}(0)\left(\left\langle m^{\prime}\left|\hat{J}_{n}^{2} \rho\right| m^{\prime}\right\rangle+c . c .\right)}+\mathcal{O}\left(\phi^{3}\right) .
$$

Using a binomial expansion of the square root for small $\phi$, we obtain

$$
\begin{aligned}
d_{H}^{2}(\phi) & \approx \frac{\phi^{2}}{4} \sum_{m}\left(\left\langle m^{\prime}\left|\hat{J}_{n}^{2} \rho\right| m^{\prime}\right\rangle+\text { c.c. }\right)+\mathcal{O}\left(\phi^{3}\right) \\
& =\frac{\phi^{2}}{2} \sum_{m}\left\langle m^{\prime}\left|\hat{J}_{n}^{2} \rho\right| m^{\prime}\right\rangle+\mathcal{O}\left(\phi^{3}\right),
\end{aligned}
$$

using the condition that the trace is cyclic. Finally, as $\left\langle\hat{J}_{n}\right\rangle=0$ (Eq. (20)), the CFI becomes

$$
\begin{aligned}
F_{c}(0) & =4 \sum_{m}\left\langle m^{\prime}\left|\hat{J}_{n}^{2} \rho\right| m^{\prime}\right\rangle \\
& =4 \Delta \hat{J}_{n}^{2} \\
& =F_{Q}
\end{aligned}
$$

where, $\Delta J_{n}^{2}$ is the variance of generator. The last equality appears since $F_{c} \leq F_{Q} \leq \Delta J_{n}^{2}$. That is, if our initial state, phase generator, and measurement basis satisfy these conditions, our measurement is guaranteed to saturate the QCRB. To illustrate this with an example, consider the case of one-axis twisting $U_{1}=\exp \left(i r_{1} J_{x}^{2}\right)$. Choosing $\hat{\Pi}$ as the parity operator in the $J_{z}$ basis, we see that $\left|\psi_{1}\right\rangle=U_{1}\left|\frac{N}{2}\right\rangle$ is a parity eigenstate. Choosing $J_{n}=J_{y}$, we see that our second condition is also met. Therefore, choosing $U_{2}=1$ (such that $\left|m^{\prime}\right\rangle=U_{2}|m\rangle=$ $|m\rangle$ ), will saturate the QCRB. However, this choice is not unique. Selecting $U_{2}$ such that the parity is conserved (that is, such that $\left|m^{\prime}\right\rangle$ is also an eigenstate of $\hat{\Pi}=\sum_{m}|m\rangle\langle m|$, or equivalently, $|m\rangle$ is an eigenstate of $\hat{\Pi}=$ $\left.\sum_{m}^{\prime}\left|m^{\prime}\right\rangle\left\langle m^{\prime}\right|\right)$, will also satisfy the conditions for optimality. An example of an IBR that satisfies this condition is $U_{2}=\exp \left(i r_{2} J_{x}^{2}\right)$. In the next section, we will examine what the optimal choice of $U_{2}$ is in order to maximise the robustness to detection noise.

\section{INTERACTION-BASED READOUT TO SATURATE THE NQCRB}

The NQCRB sets the maximum achievable CFI in the presence of detection noise $\sigma$, what remains is to find an IBR which achieves this limit. Our goal is to find $U_{2}$ such that the probability distribution $P_{m}=\left|\left\langle m\left|U_{2}\right| \psi_{\phi}\right\rangle\right|^{2}$ saturates the NQCRB. It was shown $\operatorname{in}^{57}$ that for $\phi \ll 1$, selecting $U_{2}=U_{1}^{\dagger}$ saturates the QCRB. At some value $\phi=\phi_{0}$,

$$
U_{1}^{\dagger} e^{i \hat{J}_{n} \phi_{0}} U_{1}\left|\psi_{0}\right\rangle=\frac{1}{\sqrt{2}}\left(\left|\psi_{0}\right\rangle+\left|\psi^{\prime}\right\rangle\right) \equiv\left|\psi_{b}\right\rangle
$$

where

$$
\left|\psi^{\prime}\right\rangle=\left(\hat{1}-\left|\psi_{0}\right\rangle\left\langle\psi_{0}\right|\right)\left|\psi_{b}\right\rangle / \sqrt{1-\left|\left\langle\psi_{b} \mid \psi_{0}\right\rangle\right|^{2}}
$$

We can artificially construct an IBR that is maximally robust to noise simply by constructing a unitary operator $U_{\mathrm{p}}$ that maps this state to one with distribution $P_{\mathrm{opt}}$ :

$$
U_{\mathrm{p}}=\left|\frac{N}{2}\right\rangle\left\langle\frac{N}{2}|+| \frac{-N}{2}\right\rangle\left\langle\psi^{\prime}\left|+\sum_{m=-N / 2+1}^{N / 2-1}\right| m\right\rangle\left\langle m^{\prime}\right|
$$

where $\left\{\left|m^{\prime}\right\rangle\right\}$ completes the orthogonal basis containing $\left|\frac{N}{2}\right\rangle$ and $\left|\psi^{\prime}\right\rangle$. Thus, the optimum IBR is

$$
U_{2}=U_{\mathrm{p}} U_{1}^{\dagger} \equiv U_{\mathrm{opt}}
$$




\begin{tabular}{|l||l|l|} 
Scheme: & $U_{1}$ & $r$ \\
\hline OAT & $e^{i r \hat{J}_{z}^{2}} e^{i \frac{\pi}{2} \hat{J}_{y}}$ & 0.2 \\
TACT & $e^{i r\left(\hat{J}_{x}^{2}-\hat{J}_{y}^{2}\right)}$ & 0.032 \\
TNT & $e^{i r\left(\hat{J}_{z}^{2}-\frac{N}{2} \hat{J}_{x}\right)} e^{i \frac{\pi}{2} \hat{J}_{y}}$ & 0.0715 \\
Cat & $e^{i r \hat{J}_{z}^{2}} e^{i \frac{\pi}{2} \hat{J}_{y}}$ & $\frac{\pi}{2}$ \\
QPT & $\mathcal{T} \exp \left(\frac{-i}{\hbar} \int_{0}^{t_{0}} \hat{H}\left(t^{\prime}\right) d t^{\prime}\right) e^{i \frac{\pi}{2} \hat{J}_{y}}$ &
\end{tabular}

Table 1. Details of the quantum state $\left|\psi_{1}\right\rangle=U_{1}\left|\frac{N}{2}\right\rangle$ used in Fig. (3). For TACT and TNT, $r$ was chosen to maximise $F_{Q}$ for $N=100$, while for OAT, a moderate value of $r$ was chosen such that the state was no longer in the spin-squeezed regime, ${ }^{54}$ but not sufficient to reach the maximum QFI spin-cat state, which occurs at $r=\frac{\pi}{2}$.
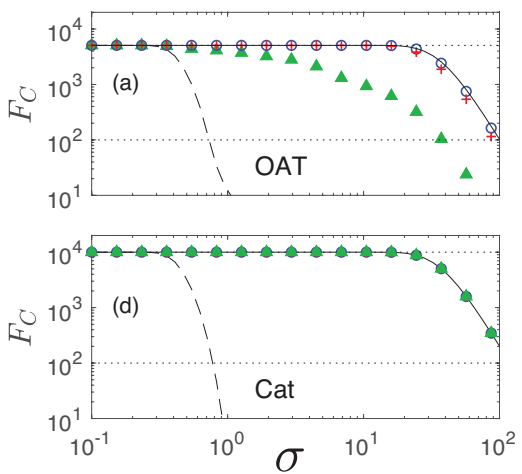
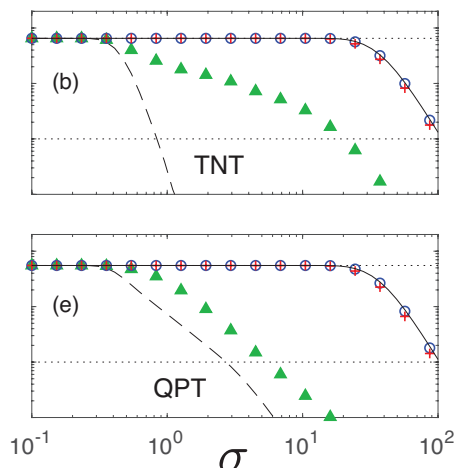
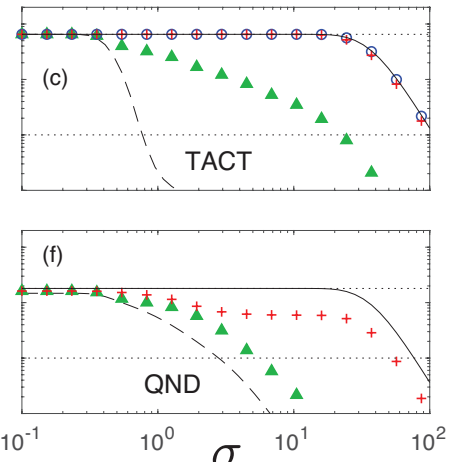

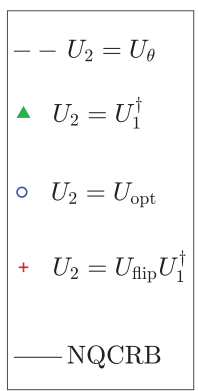

Figure 3. $F_{C}(\sigma)$ for (a): OAT with $r=0.2$, (b): TNT, (c): TACT, (d): OAT with $r=\frac{\pi}{2}$ (which corresponds to a spin-cat state), (e): QPT, and (f): QND. $U_{\theta}=e^{i \frac{\pi}{2} \hat{J}_{y}}$ for OAT, Cat, and TNT, and $U_{\theta}=1$ for TACT, QPT, and QND. The upper and lower dotted black lines indicate the QCRB $\left(F_{C}=F_{Q}\right)$ and SNL $\left(F_{C}=N\right)$, respectively. $N=100$ for all cases, and we have optimised over $\phi$. The optimum $\phi$ is close to $\phi_{0}$ for $U_{2}=U_{\text {opt }}$, and close to 0 for $U_{2}=U_{\text {flip }} U_{1}^{\dagger}$.

Fig. (3) shows the CFI calculated from $P_{m}=\left|\left\langle m\left|U_{\text {opt }}\right| \psi_{\phi}\right\rangle\right|^{2}$ after convolving with detection noise, for quantum enhanced states generated from OAT, TACT, TNT, and QPT. Details of these states are provided in table $(1)^{*}$. In all cases, we find that this IBR saturates the NQCRB. To understand the mechanism for this, we consider the effect of detection noise on the probability distributions. Fig. (4) shows $P_{m}(\phi)$ and $P_{m}(\phi+\delta \phi)$, with (right column) and without (left column) noise, for the case of OAT. When $U_{2}=U_{1}^{\dagger}$ ((a) and (e)), the change in probability is centred around $m=\frac{N}{2}$ and nearby elements. When detection noise is added, $P_{m}(\phi)$ and $P_{m}(\phi+\delta \phi)$ become less distinct as the adjacent elements are mixed. However, by applying $U_{2}=U_{\text {opt }}((\mathrm{b})$ and (f)), all of the probability in elements $m \neq \frac{N}{2}$ is transferred to $m=-\frac{N}{2}$ such that $P_{m}=P_{\text {opt }}$. We stress that the application of $U_{\mathrm{opt}}$ does not effect the CFI in the absence of noise - the Hellinger distance

$$
d_{H}^{2}=1-\sum_{m} \sqrt{P_{m}(\phi) P_{m}(\phi+\delta \phi)}
$$

is identical in (a) and (b) $\left(d_{H} \approx 0.24\right)$. However, $U_{\text {opt }}$ does affect how distinguishable the states remain after the addition of detection noise: $d_{H} \approx 0.067$, and 0.201 for (e) and (f) respectively.

\section{APPROACHING THE NQCRB WITH OAT-BASED IBRS}

While our optimum IBR gives us insight into what maximises robustness, it is of no use to us unless we can find a physical mechanism with which it can be implemented. However, we can construct an IBR which has similar properties to the ideal case with the OAT mechanism. The OAT unitary can be used to create the well known spin-cat state: ${ }^{76,77}$

$$
e^{i \frac{\pi}{2} \hat{J}_{y}^{2}}|m\rangle=\frac{1}{\sqrt{2}} e^{i \frac{\pi}{4}}\left(|m\rangle+i(-1)^{m}|-m\rangle\right) \equiv|\beta(m)\rangle,
$$

\footnotetext{
${ }^{*}$ By 'quantum-enhanced states', we mean 'states with $F_{Q}>N$ '.
} 

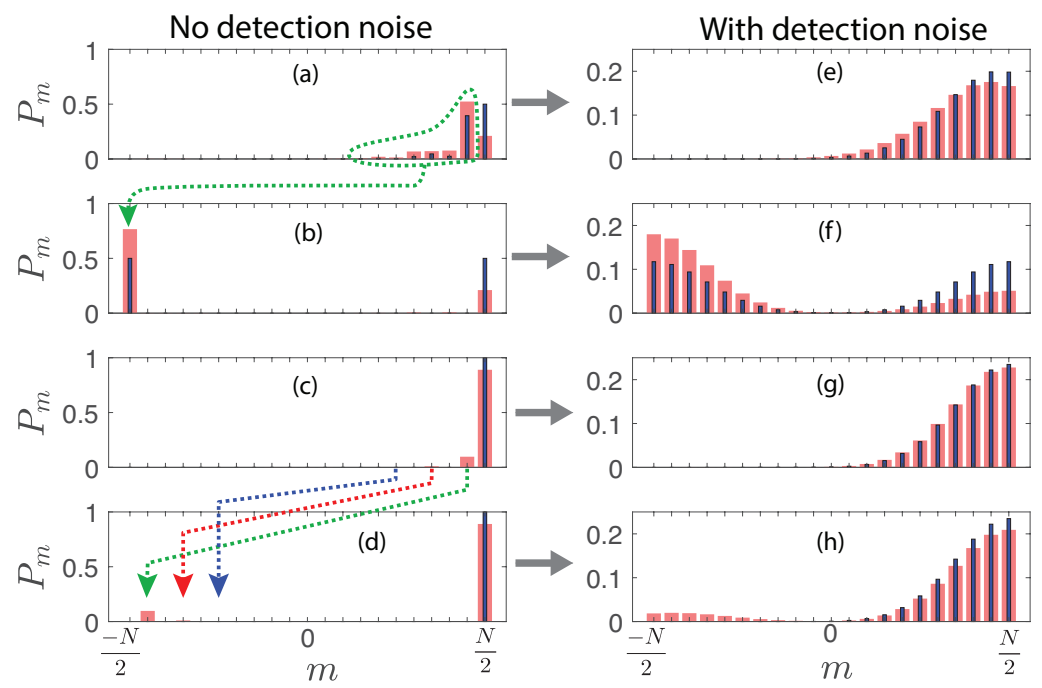

Figure 4. $\quad P_{m}(\phi)$ (blue thin bars) and $P_{m}(\phi+\delta \phi)$ (pink thick bars) with (right column) and without (left column) detection noise $\sigma$. (a) \& (e): $U_{2}=U_{1}^{\dagger}, \phi=\phi_{0}$. (b)\&(f): $U_{2}=U_{\mathrm{opt}}, \phi=\phi_{0}$. (c)\&(g): $U_{2}=U_{1}^{\dagger}, \phi=0 . \quad(\mathrm{d}) \&(\mathrm{~h})$ $U_{2}=U_{\text {flip }} U_{1}^{\dagger}, \phi=0$. The Hellinger distance $d_{H}$ is (a-d): 0.238, (e): 0.067, (f): 0.201, (g): 0.012, (h): 0.232. Parameters: $N=20, \sigma=3, r=0.2, \delta_{\phi}=\frac{1}{N}, \phi_{0}=0.118$. The behaviour of $U_{\mathrm{p}}$ and $U_{\text {flip }}$ is indicated by the arrows between (a)\&(b), and $(\mathrm{c}) \&(\mathrm{~d})$, respectively.

for even $N^{\dagger}$. This state has the unusual property that $\left|\left\langle\beta(m)\left|e^{i \frac{\pi}{2} \hat{J}_{z}}\right| \beta(m)\right\rangle\right|^{2}=\cos ^{2} \frac{m \pi}{2}$. That is, even- $m$ states are unaffected by a $\pi$ rotation, while odd- $m$ states become orthogonal. As such, a $\frac{\pi}{2}$ phase shift followed by secondary application of $e^{i \frac{\pi}{2} \hat{J}_{y}^{2}}$ will return $|\beta(m)\rangle$ to $|m\rangle$ if $m$ is even, or transfer it to an orthogonal state if $m$ is odd. Specifically

$$
e^{i \frac{\pi}{2} \hat{J}_{y}^{2}} e^{i \frac{\pi}{2} \hat{J}_{z}} e^{i \frac{\pi}{2} \hat{J}_{y}^{2}}=-\sum_{m} i^{m(m-1)}\left|-1^{m} m\right\rangle\langle m| \equiv U_{\text {flip }} .
$$

The action of $U_{\text {flip }}$ is to exchange the odd elements of $P_{m}$ with $P_{-m}$, while leaving the even elements unaffected, as illustrated in fig.(4) (d) and (h) ${ }^{\ddagger}$. For sufficiently small $\phi$, most of the CFI for the state $U_{1}^{\dagger}\left|\psi_{\phi}\right\rangle$ is usually contained in the elements $m=\frac{N}{2}$ and $m=\frac{N}{2}-1$ ((c) and (g)). Applying $U_{\text {flip }}$ to this state transfers probability from $m=\frac{N}{2}-1$ to $m=-\left(\frac{N}{2}-1\right)$, forming a distribution almost as robust as $P_{\mathrm{opt}}$.

Fig. (3) shows the performance of this scheme compared to $U_{\text {opt }}$ for quantum enhanced states generated via OAT, TACT, and TNT (see table (1)). In these three cases we see that $U_{2}=U_{\text {flip }} U_{1}^{\dagger}$ is very close to the optimum case $\left(U_{2}=U_{\text {opt }}\right.$ and the NQCRB), and achieves sensitivity very close to the QCRB for detection noise $\sigma$ significantly exceeding $\sqrt{N}$. For comparison, we have also included the previously considered case of an echo, where $U_{2}=U_{1}^{\dagger}$, which performs significantly better than the case of no IBR $\left(U_{2}=U_{\theta}\right.$, where only a linear rotation is used to maximise the CFI), but not nearly as well as $U_{2}=U_{\text {flip }} U_{1}^{\dagger}$. We have also included the special case of OAT with $r=\frac{\pi}{2}$, which corresponds to the maximum QFI spin-cat state. In this case, both $U_{2}=U_{\text {flip }} U_{1}^{\dagger}$ and $U_{2}=U_{1}^{\dagger}$ saturate the NQCRB, while the case of no IBR loses all quantum enhancement for $\sigma \lesssim 1$. The reason why there is no need for the extra application of $U_{\text {flip }}$ is because the state $U_{1}^{\dagger}\left|\psi_{\phi}\right\rangle$ already yields a probability distribution identical to $P_{\mathrm{opt}}$, and is unchanged by application of $U_{\text {flip }}$. The outstanding performance of the echo IBR for this state was first reported in $^{60}$ and subsequently in, ${ }^{61,65}$ but it was not known that this is the maximum achievable sensitivity ${ }^{\S}$.

\footnotetext{
${ }^{\dagger}$ For odd $N$ we require an additional rotation: an equal superposition cat is generated by $e^{i \frac{\pi}{2} \hat{J}_{y}} e^{i \frac{\pi}{2} \hat{J}_{y}^{2}}|m\rangle$.

${ }^{\ddagger}$ For odd $N$, an IBR that performs the same function is given by $U_{\text {flip }}=e^{i \frac{\pi}{2} \hat{J}_{y}\left(\hat{J}_{y}+1\right)} e^{i \theta \hat{J}_{z}} e^{i \frac{\pi}{2} \hat{J}_{y}\left(\hat{J}_{y}+1\right)}$, with $\theta=$ $\frac{\pi}{2}(1+1 / N)$

${ }^{\S}$ We note that ${ }^{61}$ reports higher robustness than this. However, the state is identical, and the discrepancy is due to a different convention for the detection noise
} 
We also considered QPT, where the increased QFI is generated by slowly varying the parameters in a timedependent Hamiltonian, such that the ground state is adiabatically transformed to one with high QFI. We implemented this with a Hamiltonian of the form

$$
\hat{H}=\hbar \chi\left(\hat{J}_{x} \cos ^{2} \frac{\pi}{2} \frac{t}{t_{0}}+\hat{J}_{z}^{2} \sin ^{2} \frac{\pi}{2} \frac{t}{t_{0}}\right),
$$

such that

$$
U_{1}=\mathcal{T}\left[\exp \left(\frac{-i}{\hbar} \int_{0}^{t_{0}} \hat{H}\left(t^{\prime}\right) d t^{\prime}\right)\right] e^{i \frac{\pi}{2} \hat{J}_{y}},
$$

where $\mathcal{T}$ represents the time-ordering operator. In the limit $\chi t_{0} \rightarrow \infty, U_{1}\left|\frac{N}{2}\right\rangle=|0\rangle$, the twin-Fock state. We chose a moderate value $\chi t_{0}=20$, such that the final state contains non-zero elements on either side of $m=0$. Unlike the previous examples, when making measurements on the state $U_{1}^{\dagger}\left|\psi_{\phi}\right\rangle$ for small $\phi$, most of the CFI is contained in the elements $m=\frac{N}{2}$ and $m=\frac{N}{2}-2$, such that $U_{\text {flip }}$ has little effect. This is easily rectified, however, by using a modified IBR with $U_{\text {flip }}^{\prime}=e^{i \frac{\pi}{2} \hat{J}_{y}^{2}} e^{i \frac{\pi}{4} \hat{J}_{z}} e^{i \frac{\pi}{2} \hat{J}_{y}^{2}}$, which for $N \gg 1, U_{\text {flip }}|m\rangle \approx|-m\rangle$ if $m / 2$ is odd. We see in Fig. (3e) that this IBR is very close to the NQCRB.

The benefit of our IBR is not limited to pure states. We consider a quantum enhanced mixed state

$$
\rho=\sum_{m} e^{-\frac{m^{2}}{\Delta^{2}}}|m\rangle\langle m| /\left(\sum_{m} e^{-\frac{m^{2}}{\Delta^{2}}}\right) .
$$

We chose $\Delta=1$, which corresponds to a state with significant quantum enhancement, yet is far from pure, with the purity $\gamma=\operatorname{Tr}\left[\rho^{2}\right] \approx 0.4$. Such a state may arise from quantum enhancement via a strong QND interaction with a detuned optical field, as described in, ${ }^{78}$ with an imperfect measurement leading to uncertainty in $m$. Unlike the previous states considered, this state is mixed, so there is no unitary operator that maps this distribution to $P_{\mathrm{opt}}$. However, at $\phi=0$, the final distribution is similar to the QPT case, which inspires us to use the same IBR, namely $U_{2}=U_{\text {flip }} U_{1}^{\dagger}$, with $U_{1}$ generated via the adiabatic evolution considered in the QPT example. We see in Fig. (3f) that while this case isn't as robust as previous examples, the general trend is the same, that is $U_{2}=U_{\text {flip }} U_{1}^{\dagger}$ is more robust than $U_{2}=U_{1}^{\dagger}$, which in turn outperforms $U_{2}=U_{\theta}$. As the state is mixed, we cannot systematically construct $U_{\text {opt }}$. For completeness, we have also investigated applying our IBR to states with no quantum enhancement, such as coherent spin-states,${ }^{79}$ and find qualitatively similar results.

\section{DISCUSSION}

The results of this paper may form an integral part of future quantum-enhanced sensing technologies, as highQFI states are particularly susceptible to detection noise. While OAT-based quantum enhancement schemes are not yet capable of manufacturing spin-cat states (and therefore $U_{\text {flip }}$ ), progress in this area is rapid, particularly in schemes based on optically induced non-linearities, ${ }^{6,18}$ and Rydberg atoms. ${ }^{80}$ Furthermore, we have provided insight and a systematic approach for constructing a robust IBR. Armed with this insight, schemes that approximate our optimum scheme may be found through other dynamical mechanisms that are perhaps easier to implement in a particular system. For example, it has been shown that QPT can be used to engineer spin-cat states, ${ }^{46}$ so could potentially be used to construct a near-optimum IBR. One might question the wisdom of using an IBR that requires the ability to create a maximum QFI cat state in cases where the QFI of the input state is less than this. However, there may be situations when it is impractical to use a state preparation capable of creating a cat state, such as when the preparation time is limited. ${ }^{63}$ Similarly, a state with less quantum enhancement may be desirable in the presence of external phase noise. In these situations, the presence of unavoidably large detection noise will still necessitate the use of a high-performance IBR in order to achieve high sensitivity. Finally, the NQCRB provides a limit for the performance of all IBR's. Once the sensitivity approaches this limit, further gains can only be made through the reduction of detection noise, rather than via improvement of the IBR.

\section{ACKNOWLEDGMENTS}

We acknowledge fruitful discussions with Samuel Nolan, Safoura Mirkhalaf, Luca Pezze, Augusto Smerzi, Manuel Gessner, and Jacob Dunningham. RL is supported by an Australian Government Research Training Program (RTP) Scholarship. This work was supported by Australian Research Council Discovery Project DP190101709. 


\section{REFERENCES}

[1] Cronin, A. D., Schmiedmayer, J., and Pritchard, D. E., "Optics and interferometry with atoms and molecules," Rev. Mod. Phys. 81, 1051-1129 (Jul 2009).

[2] Pezze, L., Smerzi, A., Oberthaler, M. K., Schmied, R., and Treutlein, P., "Quantum metrology with nonclassical states of atomic ensembles," arXiv:1609.01609 (2016).

[3] Esteve, J., Gross, C., A., W., Giovanazzi, S., and Oberthaler, M. K., "Squeezing and entanglement in a Bose-Einstein condensate," Nature 455(7217), 1216 (2008).

[4] Appel, J., Windpassinger, P. J., Oblak, D., Hoff, U. B., Kjaergaard, N., and Polzik, E. S., "Mesoscopic atomic entanglement for precision measurements beyond the standard quantum limit," Proceedings of the National Academy of Sciences 106(27), 10960-10965 (2009).

[5] Leroux, I. D., Schleier-Smith, M. H., and Vuletić, V., "Implementation of cavity squeezing of a collective atomic spin," Phys. Rev. Lett. 104, 073602 (Feb 2010).

[6] Schleier-Smith, M. H., Leroux, I. D., and Vuletić, V., "Squeezing the collective spin of a dilute atomic ensemble by cavity feedback," Phys. Rev. A 81, 021804 (Feb 2010).

[7] Schleier-Smith, M. H., Leroux, I. D., and Vuletić, V., "States of an ensemble of two-level atoms with reduced quantum uncertainty," Phys. Rev. Lett. 104, 073604 (Feb 2010).

[8] Gross, C., Zibold, T., Nicklas, E., Estève, J., and Oberthaler, M. K., "Nonlinear atom interferometer surpasses classical precision limit," Nature 464, 1165-1169 (04 2010).

[9] Riedel, M. F., Böhi, P., Li, Y., Hänsch, T. W., Sinatra, A., and Treutlein, P., "Atom-chip-based generation of entanglement for quantum metrology," Nature 464, 1170-1173 (04 2010).

[10] Lücke, B., Scherer, M., Kruse, J., Pezze, L., Deuretzbacher, F., Hyllus, P., Topic, O., Peise, J., Ertmer, W., Arlt, J., Santos, L., Smerzi, A., and Klempt, C., "Twin matter waves for interferometry beyond the classical limit," Science 334, 773-776 (11 2011).

[11] Hamley, C. D., Gerving, C. S., Hoang, T. M., Bookjans, E. M., and Chapman, M. S., "Spin-nematic squeezed vacuum in a quantum gas," Nat Phys 8, 305-308 (04 2012).

[12] Berrada, T., van Frank, S., Bücker, R., Schumm, T., Schaff, J. F., and Schmiedmayer, J., "Integrated Mach-Zehnder interferometer for Bose-Einstein condensates," Nature Communications 4, 2077 EP - (06 2013).

[13] Ockeloen, C. F., Schmied, R., Riedel, M. F., and Treutlein, P., "Quantum metrology with a scanning probe atom interferometer," Phys. Rev. Lett. 111, 143001 (Oct 2013).

[14] Strobel, H., Muessel, W., Linnemann, D., Zibold, T., Hume, D. B., Pezzè, L., Smerzi, A., and Oberthaler, M. K., "Fisher information and entanglement of non-gaussian spin states," Science 345(6195), 424-427 (2014).

[15] Muessel, W., Strobel, H., Linnemann, D., Hume, D. B., and Oberthaler, M. K., "Scalable spin squeezing for quantum-enhanced magnetometry with bose-einstein condensates," Phys. Rev. Lett. 113, 103004 (Sep 2014).

[16] Muessel, W., Strobel, H., Linnemann, D., Zibold, T., Julia-Diaz, B., and Oberthaler, M. K., "Twist-andturn spin squeezing in Bose-Einstein condensates," arXiv:150\%.02930 (2015).

[17] Kruse, I., Lange, K., Peise, J., Lücke, B., Pezzè, L., Arlt, J., Ertmer, W., Lisdat, C., Santos, L., Smerzi, A., and Klempt, C., "Improvement of an atomic clock using squeezed vacuum," Phys. Rev. Lett. 117, 143004 (Sep 2016).

[18] Hosten, O., Engelsen, N. J., Krishnakumar, R., and Kasevich, M. A., "Measurement noise 100 times lower than the quantum-projection limit using entangled atoms," Nature 529, 505 EP - (01 2016).

[19] Zou, Y.-Q., Wu, L.-N., Liu, Q., Luo, Lu, X.-Y., Guo, S.-F. G., Cao, J.-H., Tey, and Meng Khoon, You, L., "Beating the classical precision limit with spin-1 Dicke state of more than 10000 atoms," arXiv:1802.10288 (2018).

[20] Hyllus, P., Gühne, O., and Smerzi, A., "Not all pure entangled states are useful for sub-shot-noise interferometry," Phys. Rev. A 82, 012337 (Jul 2010).

[21] Hyllus, P., Laskowski, W., Krischek, R., Schwemmer, C., Wieczorek, W., Weinfurter, H., Pezzé, L., and Smerzi, A., "Fisher information and multiparticle entanglement," Phys. Rev. A 85, 022321 (Feb 2012).

[22] Tóth, G., "Multipartite entanglement and high-precision metrology," Phys. Rev. A 85, 022322 (Feb 2012). 
[23] Agarwal, G. S. and Puri, R. R., "Cooperative behavior of atoms irradiated by broadband squeezed light," Phys. Rev. A 41, 3782-3791 (Apr 1990).

[24] Kuzmich, A., Mølmer, K., and Polzik, E., "Spin squeezing in an ensemble of atoms illuminated with squeezed light," Phys. Rev. Lett. 79, 4782-4785 (Dec 1997).

[25] Moore, M. G., Zobay, O., and Meystre, P., "Quantum optics of a Bose-Einstein condensate coupled to a quantized light field," Phys. Rev. A 60, 1491-1506 (Aug 1999).

[26] Jing, H., Chen, J.-L., and Ge, M.-L., "Quantum-dynamical theory for squeezing the output of a BoseEinstein condensate," Phys. Rev. A 63, 015601 (Nov 2000).

[27] Fleischhauer, M. and Gong, S., "Stationary source of nonclassical or entangled atoms," Phys. Rev. Lett. 88, 070404 (Jan 2002).

[28] Haine, S. A. and Hope, J. J., "Outcoupling from a Bose-Einstein condensate with squeezed light to produce entangled-atom laser beams," Phys. Rev. A 72, 033601 (Sep 2005).

[29] Haine, S. A. and Hope, J. J., "A multi-mode model of a non-classical atom laser produced by outcoupling from a Bose-Einstein condensate with squeezed light," Laser Physics Letters 2(12), 597-602 (2005).

[30] Haine, S. A., Olsen, M. K., and Hope, J. J., "Generating controllable atom-light entanglement with a Raman atom laser system," Phys. Rev. Lett. 96, 133601 (Apr 2006).

[31] Szigeti, S. S., Tonekaboni, B., Lau, W. Y. S., Hood, S. N., and Haine, S. A., "Squeezed-light-enhanced atom interferometry below the standard quantum limit," Phys. Rev. A 90, 063630 (Dec 2014).

[32] Haine, S. A., Szigeti, S. S., Lang, M. D., and Caves, C. M., "Heisenberg-limited metrology with information recycling," Phys. Rev. A 91, 041802 (Apr 2015).

[33] Kuzmich, A., Bigelow, N. P., and Mandel, L., "Atomic quantum non-demolition measurements and squeezing," Europhys. Lett. 42(5), 481-486 (1998).

[34] Kuzmich, A., Mandel, L., and Bigelow, N. P., "Generation of spin squeezing via continuous quantum nondemolition measurement," Phys. Rev. Lett. 85, 1594-1597 (Aug 2000).

[35] Louchet-Chauvet, A., Appel, J., Renema, J. J., Oblak, D., Kjaergaard, N., and Polzik, E. S., "Entanglementassisted atomic clock beyond the projection noise limit," New Journal of Physics 12(6), 065032 (2010).

[36] Hammerer, K., Sørensen, A. S., and Polzik, E. S., "Quantum interface between light and atomic ensembles," Rev. Mod. Phys. 82, 1041-1093 (Apr 2010).

[37] Duan, L.-M., Sørensen, A., Cirac, J. I., and Zoller, P., "Squeezing and entanglement of atomic beams," Phys. Rev. Lett. 85, 3991-3994 (Nov 2000).

[38] $\mathrm{Pu}, \mathrm{H}$. and Meystre, P., "Creating macroscopic atomic Einstein-Podolsky-Rosen states from Bose-Einstein condensates," Phys. Rev. Lett. 85, 3987-3990 (Nov 2000).

[39] Nolan, S. P., Sabbatini, J., Bromley, M. W. J., Davis, M. J., and Haine, S. A., "Quantum enhanced measurement of rotations with a spin-1 Bose-Einstein condensate in a ring trap," Phys. Rev. A 93, 023616 (Feb 2016).

[40] Kitagawa, M. and Ueda, M., "Squeezed spin states," Phys. Rev. A 47, 5138-5143 (Jun 1993).

[41] Sørensen, A. S. and Mølmer, K., "Entanglement and extreme spin squeezing," Phys. Rev. Lett. 86, 44314434 (May 2001).

[42] Haine, S. A., Lau, J., Anderson, R. P., and Johnsson, M. T., "Self-induced spatial dynamics to enhance spin squeezing via one-axis twisting in a two-component Bose-Einstein condensate," Phys. Rev. A 90, 023613 (Aug 2014).

[43] Ma, J. and Wang, X., "Fisher information and spin squeezing in the Lipkin-Meshkov-Glick model," Phys. Rev. A 80, 012318 (Jul 2009).

[44] Law, C. K., Ng, H. T., and Leung, P. T., "Coherent control of spin squeezing," Phys. Rev. A 63, 055601 (Apr 2001).

[45] Lee, C., "Adiabatic mach-zehnder interferometry on a quantized Bose-Josephson junction," Phys. Rev. Lett. 97, 150402 (Oct 2006).

[46] Lee, C., "Universality and anomalous mean-field breakdown of symmetry-breaking transitions in a coupled two-component Bose-Einstein condensate," Phys. Rev. Lett. 102, 070401 (Feb 2009).

[47] Zhang, Z. and Duan, L.-M., "Generation of massive entanglement through an adiabatic quantum phase transition in a spinor condensate," Phys. Rev. Lett. 111, 180401 (Oct 2013). 
[48] Xing, H., Wang, A., Tan, Q.-S., Zhang, W., and Yi, S., "Heisenberg-scaled magnetometer with dipolar spin-1 condensates," Phys. Rev. A 93, 043615 (Apr 2016).

[49] Luo, X.-Y., Zou, Y.-Q., Wu, L.-N., Liu, Q., Han, M.-F., Tey, M. K., and You, L., "Deterministic entanglement generation from driving through quantum phase transitions," Science 355(6325), 620-623 (2017).

[50] Feldmann, P., Gessner, M., Gabbrielli, M., Klempt, C., Santos, L., Pezzè, L., and Smerzi, A., "Interferometric sensitivity and entanglement by scanning through quantum phase transitions in spinor Bose-Einstein condensates," Phys. Rev. A 97, 032339 (Mar 2018).

[51] Huang, J., Zhuang, M., and Lee, C., "Non-Gaussian precision metrology via driving through quantum phase transitions," Phys. Rev. A 97, 032116 (Mar 2018).

[52] Demkowicz-Dobrzanski, R., Kolodynski, J., and Guta, M., "The elusive Heisenberg limit in quantumenhanced metrology," Nat Commun 3, 1063 (09 2012).

[53] Demkowicz-Dobrzanski, R., Jarzyna, M., and Kolodynski, J., "Quantum limits in optical interferometry," Progress in Optics 60, 345 (2015).

[54] Davis, E., Bentsen, G., and Schleier-Smith, M., "Approaching the Heisenberg limit without single-particle detection," Phys. Rev. Lett. 116, 053601 (Feb 2016).

[55] Hosten, O., Krishnakumar, R., Engelsen, N. J., and Kasevich, M. A., "Quantum phase magnification," Science 352(6293), 1552-1555 (2016).

[56] Fröwis, F., Sekatski, P., and Dür, W., "Detecting large quantum Fisher information with finite measurement precision," Phys. Rev. Lett. 116, 090801 (Mar 2016).

[57] Macrì, T., Smerzi, A., and Pezzè, L., "Loschmidt echo for quantum metrology," Phys. Rev. A 94, 010102 (Jul 2016).

[58] Linnemann, D., Strobel, H., Muessel, W., Schulz, J., Lewis-Swan, R. J., Kheruntsyan, K. V., and Oberthaler, M. K., "Quantum-enhanced sensing based on time reversal of nonlinear dynamics," Phys. Rev. Lett. 117, 013001 (Jun 2016).

[59] Szigeti, S. S., Lewis-Swan, R. J., and Haine, S. A., "Pumped-up SU(1,1) interferometry," Phys. Rev. Lett. 118, 150401 (Apr 2017).

[60] Nolan, S. P., Szigeti, S. S., and Haine, S. A., "Optimal and robust quantum metrology using interactionbased readouts," Phys. Rev. Lett. 119, 193601 (Nov 2017).

[61] Fang, R., Sarkar, R., and Shahriar, S., "Enhancing sensitivity of an atom interferometer to the Heisenberg limit using increased quantum noise," arXiv:1707.08260 (2018).

[62] Anders, F., Pezzè, L., Smerzi, A., and Klempt, C., "Phase magnification by two-axis countertwisting for detection-noise robust interferometry," Phys. Rev. A 97, 043813 (Apr 2018).

[63] Hayes, A. J., Dooley, S., Munro, W. J., Nemoto, K., and Dunningham, J., "Making the most of time in quantum metrology: concurrent state preparation and sensing," Quantum Science and Technology 3(3), 035007 (2018).

[64] Mirkhalaf, S. S., Nolan, S. P., and Haine, S. A., "Robustifying twist-and-turn entanglement with interactionbased readout," Phys. Rev. A 97, 053618 (May 2018).

[65] Huang, J., Zhuang, M., Lu, B., Ke, Y., and Lee, C., "Achieving heisenberg-limited metrology with spin cat states via interaction-based readout," Phys. Rev. A 98, 012129 (Jul 2018).

[66] Lewis-Swan, R. J., Norcia, M. A., Cline, J. R. K., Thompson, J. K., and Rey, A. M., "Robust spin squeezing via photon-mediated interactions on an optical clock transition," Phys. Rev. Lett. 121, 070403 (Aug 2018).

[67] Haine, S. A., "Using interaction-based readouts to approach the ultimate limit of detection-noise robustness for quantum-enhanced metrology in collective spin systems," Phys. Rev. A 98, 030303 (Sep 2018).

[68] Holland, M. J. and Burnett, K., "Interferometric detection of optical phase shifts at the Heisenberg limit," Phys. Rev. Lett. 71, 1355-1358 (Aug 1993).

[69] Giovannetti, V., Lloyd, S., and Maccone, L., "Quantum metrology," Phys. Rev. Lett. 96, 010401 (Jan 2006).

[70] Gabbrielli, M., Pezzè, L., and Smerzi, A., "Spin-mixing interferometry with Bose-Einstein condensates," Phys. Rev. Lett. 115, 163002 (Oct 2015).

[71] Yurke, B., McCall, S. L., and Klauder, J. R., "SU(2) and SU(1,1) interferometers," Phys. Rev. A 33, 4033-4054 (Jun 1986). 
[72] Pezzé, L. and Smerzi, A., "Ultra sensitive two-mode interferometry with single-mode number squeezing," Phys. Rev. Lett. 110, 163604 (Apr 2013).

[73] Wootters, W. K., "Statistical distance and hilbert space," Phys. Rev. D 23, 357-362 (Jan 1981).

[74] Braunstein, S. L. and Caves, C. M., "Statistical distance and the geometry of quantum states," Phys. Rev. Lett. 72, 3439-3443 (May 1994).

[75] Lee, J., [Introduction to Smooth Manifolds], Springer-Verlag, New York, 2 ed. (2012).

[76] Agarwal, G. S., Puri, R. R., and Singh, R. P., "Atomic Schrödinger cat states," Phys. Rev. A 56, 2249-2254 (Sep 1997).

[77] Nolan, S. P. and Haine, S. A., "Quantum Fisher information as a predictor of decoherence in the preparation of spin-cat states for quantum metrology," Phys. Rev. A 95, 043642 (Apr 2017).

[78] Haine, S. A. and Szigeti, S. S., "Quantum metrology with mixed states: When recovering lost information is better than never losing it," Phys. Rev. A 92, 032317 (Sep 2015).

[79] Radcliffe, J. M., "Some properties of coherent spin states," Journal of Physics A: General Physics 4(3), 313 (1971).

[80] Busche, H., Huillery, P., Ball, S. W., Ilieva, T., Jones, M. P. A., and Adams, C. S., "Contactless nonlinear optics mediated by long-range Rydberg interactions," Nature Physics 13, 655 EP - (03 2017). 